\title{
Examination of Application and Function of Two-phase Pumps
}

\author{
Maryam Khalili ${ }^{*}$, Akbar Alidadi Shamsabadi ${ }^{1}$, Mahdi Mohammadi Janaki ${ }^{2}$ and \\ Daryosh Mohammadi Janaki ${ }^{2}$ \\ 'Islamic Azad University, Shahrekord Branch, Department of Mechanical Engineering, \\ Shahrekord, Iran; maryam.khalili2010@gmail.com \\ ${ }^{2}$ Islamic Azad University, Najafabad Branch, Department of Industrial Engineering, Iran
}

\begin{abstract}
In different industries, a lot of money has been spent for seperation or matching of two phases of gas and liquid in order to transfer them from one place to another. In this paper, different kinds of two-phase pumps were introduced; their function methods were studied in short; as well as each pump's scope, advantages and disadvantages were discussed. Finally, all effective parameters on a two-phase pumps- spiral pump and their impact were explained. This will help to design for an intended use based on their application type and effect.
\end{abstract}

Keywords: Effective Parameters, Function Method, Two-phase Fluid of Liqiud-gas, Two-phase Pump, Spiral Pump

\section{Introduction}

The two-phase pumps, which increase the pressure of two-phase flow of liquid and gas, have been paid much attention in recent decades. The advantages include the ability to increase the flow pressure in two-phase mode with high volume of gas, work in low speed and separate the two phases of gas and liquid. These benefits lead to high industrial application of these pumps and eventually become stronger and more frugal pumps than the past. If two phases of the flow are of the same kind, the twophase flow can be changed into a one-phase flow and then transferred by changing the temperature and pressure. If not, they can be transfered by seperation from each other. Some people, including Cooper ${ }^{1}$, Murakami and Minemura $^{2}$ and others, tried to superpose the measured information with the obtained parameters but it was futile for finding their effect on pumps functions because they indicated comparison between identical conditions.
It does not mean that these findings were useless and, a lot of fruitful information was obtained from them. Therefore, all academic and non-academic research done on two-phase pumps were about identification of the effective parameters on the pump function. In this paper, advantages, disadvantages, working and application method of two-phase pumps were introduced completely and the effective parameters on pumps and their impact on flow and head of one kind of pump was investigated.

\section{Types of Two-phase Pumps}

\subsection{Two-phase Pumps of Centrifugal ${ }^{3}$}

Centrifugal pump is a most common pump used in industries, agriculture, domestic applications ${ }^{4}$, extensively for hydraulic transportation of liquids over short to medium distance through pipelines where the requirements of head and discharge are moderate ${ }^{5}$. The main structure of

${ }^{*}$ Author for correspondence 
these pumps is formed by a set of blade put on an axis and the flow parallel to it enters it and exists vertically. The working base of centrifugal pumps is due to using centrifugal forces. Every substance which is moved in a circular direction, is affected by a centrifugal force. Its orientation always tends to draw the substance of the axis or rotation center. The rotated piece inside the pump's casing leads to movement of flow; as a result, it is affected by centrifugal force and exits from the external channel. As a result of creating a relative vacuum, the atmosphere pressure causes that the flow enter the pump's body. Until the twophase flow is in the pump, the above order is repeated. The rotated piece inside the centrifugal pumps is named blade. The entrance channel is located in the center of the blade and the exit one around the blade's body. (Fig. 1)

The interruption mechanism can be summerized as follows:

Accumulation of gas in channels of the blade, separation of the fluid, and the asymmetric loop of gas in the loosenes between the blade and casing.

\subsection{Positive Displacement Pumps}

\subsubsection{Screw Pump}

The screw pump has been used as a two-phase pump for a long time, especially for transferring the two-phase flow with high amount of gas and in variable entrance conditions. In particular conditions, this pump works up to $100 \%$. Fig. 2, shows a profile of the screw pump. The multiple-phase mixture enters the pump from one side and is divided into two fluids and enter the entrances put in two sides of the pump. The volume flow depends on the pitch and diameter of the screws and the rotation velocity. When the gas is compacted, a little amount of the liquid returns to the back from the empty spaces between the screws and the shield wall which leads to reduction of the voluminous yield of the pump.The double-flow screw pump (up to about 30 bar (450 psig)) is available as a

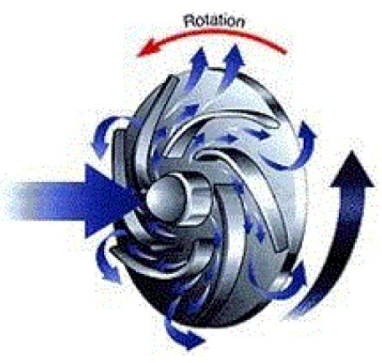

Figure 1. A semi-open blade of the centrifugal pump. low-pressure pump and up to pressure difference about $90 \mathrm{bar}(1300 \mathrm{psig})$ as a high pressure pump. According the parameters, it can have flow up to $2000 \mathrm{~m}^{3} / \mathrm{h}$.

\subsubsection{Progressing Cavity Pump (PCP)}

The PCP is made of a rubber stator and a metal rotating rotor (Figure 3). This pump, for low flow (less than 200 $\mathrm{m} 3 / \mathrm{h}$ ) and the low pressure of the outlet up to $400 \mathrm{psig}$ has a special ability for bearing the considerable amounts of the solid particles (sand).

The two spiral interior stators are made of a hard material resistant to erosion. When the rotor rotates in the stator, some holes are made which move from the suction part to the end of propulsion part and transfer the pumping materials (Figure 4). Progressing Cavity Pumping is being more and more used in oil production, mainly in (heavy oil) fields, due to its numerous technical advantages ${ }^{7}$.

Continual seal off between the spirals of rotor and stator keeps the flow stable movement in a fix flow

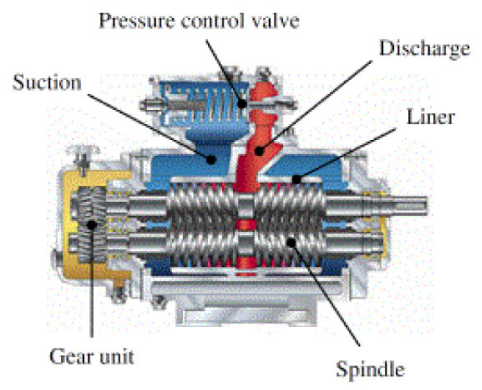

Figure 2. Sectional drawing of a double-flow screw pump ${ }^{6}$.

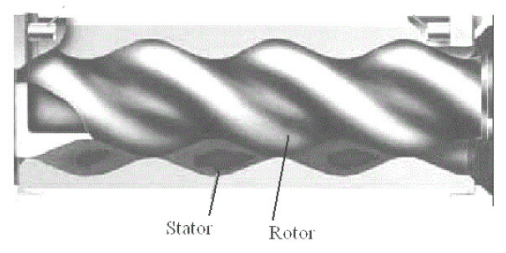

Figure 3. Rotor and stator of the PCP.

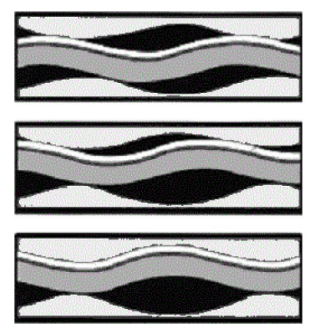

Figure 4. Working method of PCP. 
proportionate with the pump rotative velocity. However, PCP has a low ability to transfer the high amount of gas in liquid which is resulted from the limitations of the stator elastomeric materials .In Fig. 5, a model of a complete system of PCP is shown.

\subsubsection{Piston Pumps}

One on the simplest forms of the multi-phase pumps is using a big two-phase piston for compacting the mixture of gas-liquid. This method is used for the scopes of low and average flow having the maximum capacity about $110000 \mathrm{bbl} / \mathrm{d}$ (the total volume of gas and liquid) and the most outlet pressure about $1400 \mathrm{psig}$. Some models of piston pomps are shown is Fig. 6 \& 7 .

\subsection{Rotary Pump}

\subsubsection{Helicon-axial Pump}

In this pump, the flow runs in a set of pump's layer vertically. Every layer includes a helically-like rotating blade and a fix diffuser ${ }^{8}$ (Figure 8).

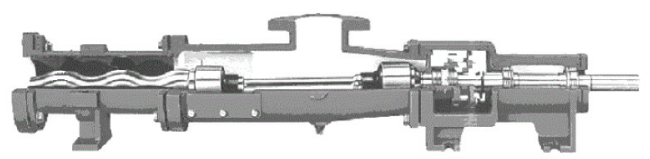

Figure 5. A model of a complete system of PCP.

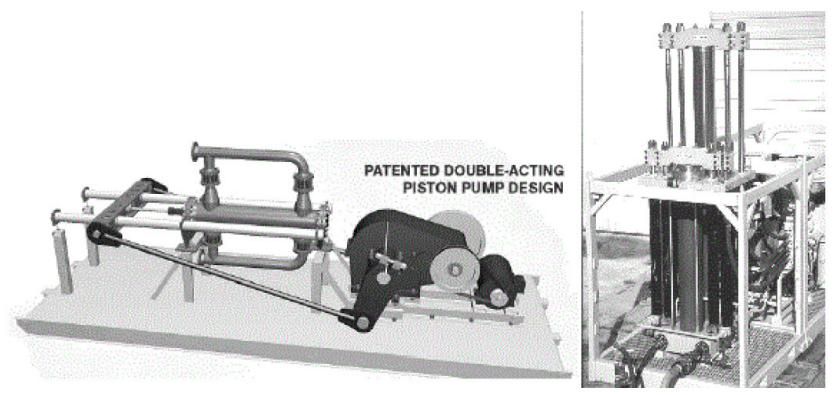

Figure $6 \& 7$. Two models of a two-phase piston pump.

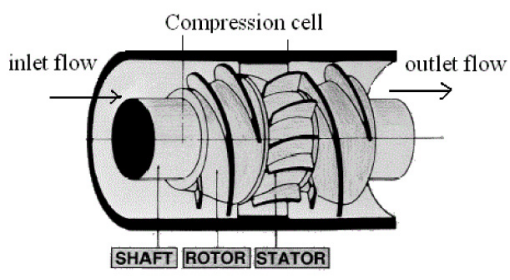

Figure 8. Inner structure of the Helicon-axial pump.
Compacting of gas in consecutive layers leads to changing the geometry of the diffuser blade to be compatible with flow reduction (Figure 9).

The advantage and disadvantages of Helicon-axial pump are listed in Table 1.

\subsubsection{The Spiral Two-phase Pump}

The different parts of this pump are shown in the Figure 10.

\section{Benefits:}

- The two-phase flow (gas-liquid) pumping

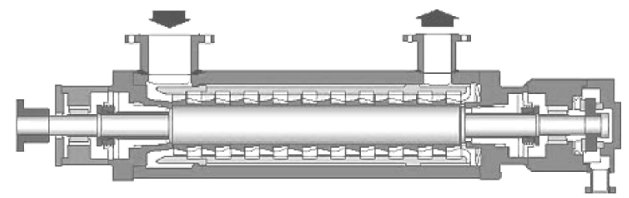

Figure 9. A complete model of a Helicon-axial pump.

Table 1. The advantage and disadvantages of Helicon-axial pump

\begin{tabular}{|c|c|}
\hline \multicolumn{2}{|c|}{ Helico-Axial pumps } \\
\hline advantages & disadvantages \\
\hline $\begin{array}{l}\text { High capacity ( } 50,000 \mathrm{bbl} / \mathrm{d} \text { to } \\
450,000 \mathrm{bbl} / \mathrm{d}) \text { and }\end{array}$ & High shear \\
\hline $\begin{array}{l}\text { Good operational range and } \\
\text { flexibility due to high speed range }\end{array}$ & Slugging problem \\
\hline $\begin{array}{l}\text { High differential pressure }(<2900 \\
\text { psig) and high pressure rise }\end{array}$ & $\begin{array}{l}\text { Not a good choice } \\
\text { for viscous fluids }\end{array}$ \\
\hline Self-adapting to flow changes & $\begin{array}{l}\text { Not a good choice } \\
\text { for low flow rates }\end{array}$ \\
\hline $\begin{array}{l}\text { Series and parallel operation is } \\
\text { possible }\end{array}$ & $\begin{array}{l}\text { Not able to operate } \\
\text { in low suction } \\
\text { pressure }\end{array}$ \\
\hline \multicolumn{2}{|l|}{$70 \mathrm{~mm}<$ Impeller diameter $<400 \mathrm{~mm}$} \\
\hline $\begin{array}{l}\text { Low potential for erosion in case of } \\
\text { solid handling }\end{array}$ & \\
\hline
\end{tabular}

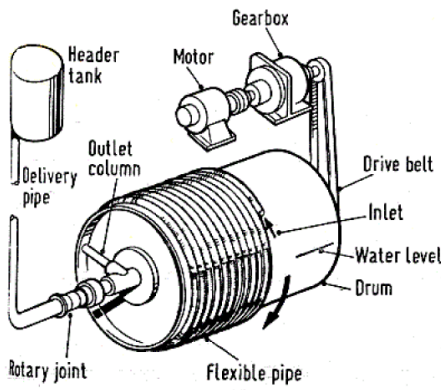

Figure 10. Shematic picture of the spiral pump. 
The spiral pump has the ability of pumping the twophase flow with density coefficient of gas in liquid up tp 95\%.

- It can used as a device to separate the gas phase from liquid one

- using wind energy

The spiral pump can also work with low rotating speed and provide the least flow for a specific height. Therefore, it is a very suitable device for coupling with wind turbine in order to transfer water.

- Pumping the river water

Using the spiral pump beside the rivers to transfer the water to the higher hights is very suitable.

- The cheap and easy constructing, installation and launching

- The plastic materials used in making this pump

When the passing flow leads to corrosion of metals, all of pieces used in this pump which are in contact with the flow can be made by plastic materials..

\section{Effective Parameters on Performance of Screw Biphasic Pump}

The carried out experiments and examination of the theoretical relations of the two-phase flow revealed that the effective parameters on this pump function are as following

\subsection{The Ratio of Liquid to the Inlet Gas (g)}

The ratio of liquid to the inlet gas in every rotation depends on the amount of the angle upon which the head of the spiral pipe moves in liquid or gas.

Effect $g$ of on the flow of the fluid: as it is shown in Figure 11, increasing the amount of liquid leads to increasing the outlet flow of the pump.

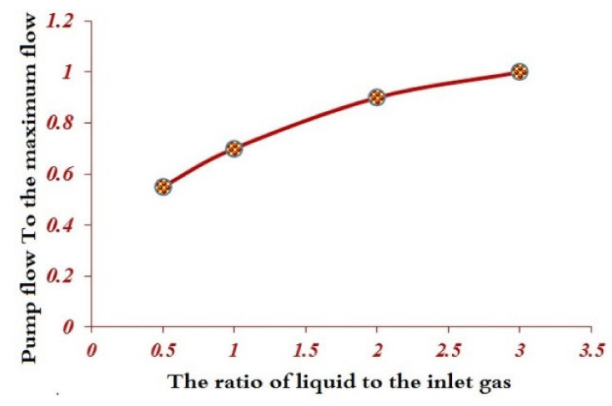

Effect of $g$ on the head: in Figure 12, the effect of ratio of liquid to the inlet gas on the head is shown. Until the ratio of liquid to the inlet gas equals one, the pump head will be increased and, when this ratio is increased, the head ratio will be reduced.

\subsection{Effect of the Rotative Velocity (N)}

Effect of the rotative velocity on flow: as it is shown in Figure 13, the increase of the rotative velocity has a direct relationship with the pump flow.

Effect of the rotative velocity on the head: according to Fig. 14, when the rotative velocity is increased, at first the amount of head is increased and when the rotative velocity reaches to maximum 1 , it is reduced.

\subsection{Effect of the Angle of the Pump Cylinder Pivot with the Horizontal}

The findings of Figure 15 reveal that when the angle of the pump cylinder pivot with the horizon is changed, the change of the flow is not tangible.

\subsection{The Pitch of the Spiral Pipe}

Changing the pitch of the spiral pipe has no remarkable effect on the pump function because the changes of the parameter of rotation in the spiral pump are not tangible.

\subsection{Number of the Rotations of the Spiral Pipe}

Number of the coils has no significant effect on the outlet flow of the pump in a specified height, but this parameter is a decisive factor for the maximum amount of the pump head because increasing the number of the coils leads to increasing the maximum amount of the head as it is shown in Fig. 16.

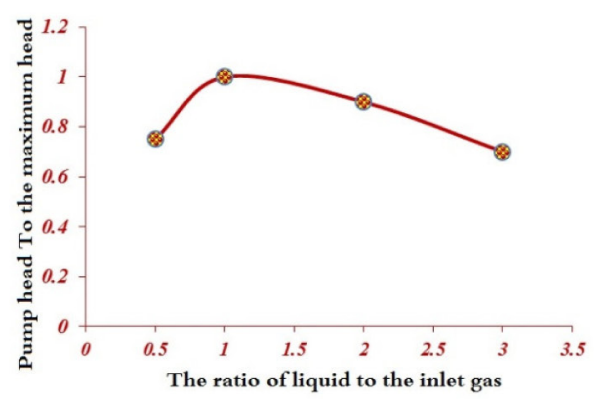

Figure $11 \& 12$. Effect of the ratio of liquid to the inlet gas on the head of the pump and flow. 

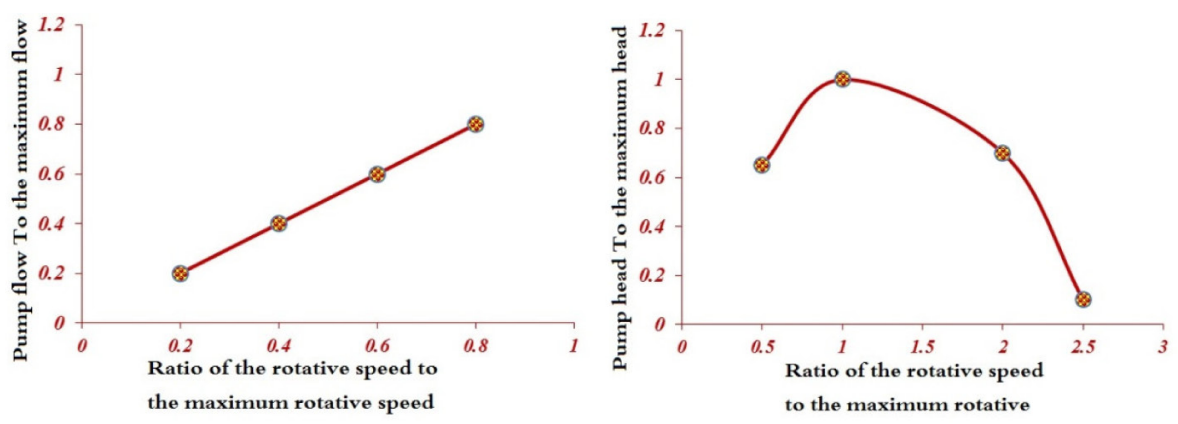

Figure $13 \& 14$. SEffect of the rotative velocity on the pump flow and head.

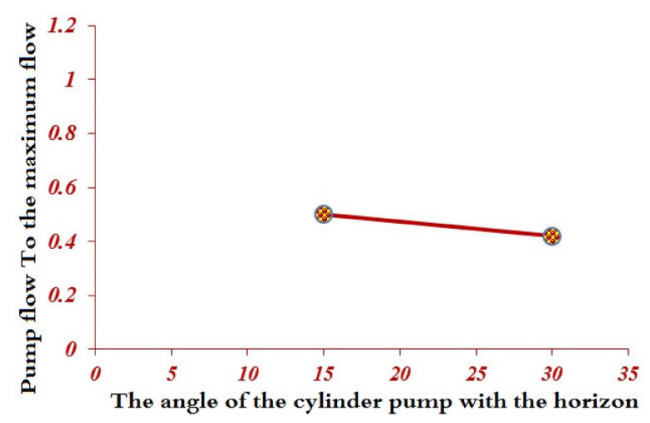

Figure 15. Effect of the angle of the cylinder pump with the horizon on the pump flow.

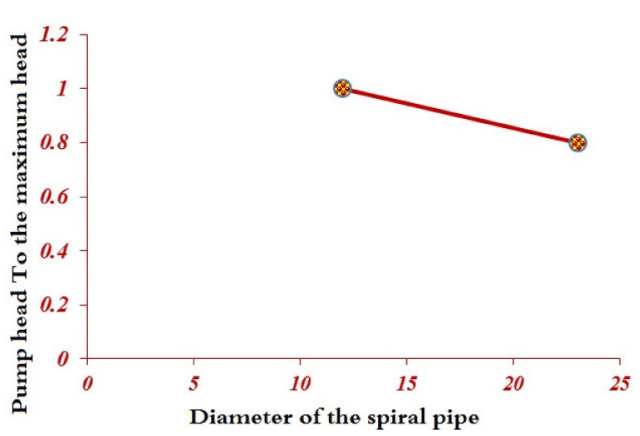

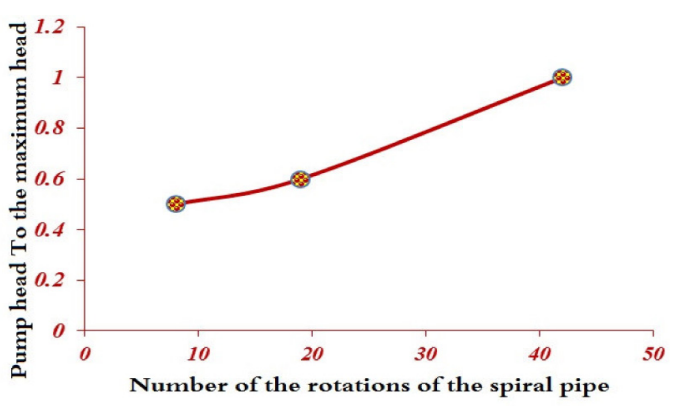

Figure 16. Effect of the number of the rotations of the spiral pipe on the pump head.

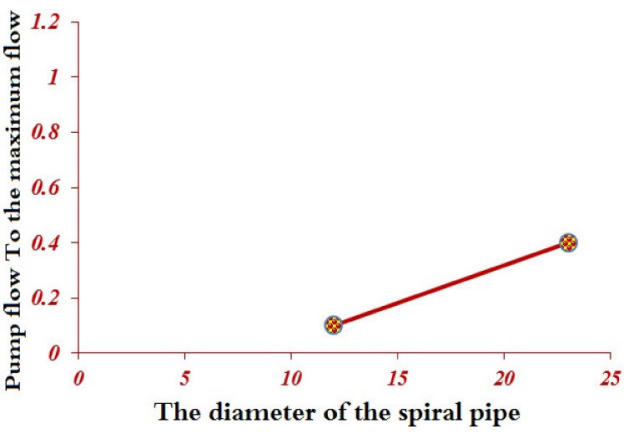

Figure 17 \& 18. Effect of the diameter of the spiral pipe on the pump flow and head.

\subsection{Diameter of the Spiral Pipe (d)}

Diameter of the spiral pipe is the determinant of the inlet flow amount to the pipe. The more the pipe diameter, the big the inlet mouth of liquid to the pipe. As a result, increasing the diameter of the spiral pipe leads to increasing the amount of the pump flow, in addition, increasing the diameter of the spiral pipe lead to reduction of the maximum amount of the head (Figures 17 \& 18).

\subsection{The Cylinder Diameter}

Increasing the cylinder diameter leads to increasing the amount of the inlet liquid in every rotation. In a fix rotation speed, when the cylinder diameter is increased, the distance which passed by the spiral pipe in liquid is increased too. In every rotation, a more volume liquid enters to the pipe and moves toward the outlet part.

\section{Conclusion}

The two-phase pumps work in the scope of rotation velocity and special pressures and the kind of pump selected is based on the intended application. Different parameters including $\mathrm{D}, \mathrm{d}, \mathrm{n}, \mathrm{P}, \mathrm{g}$ have effect on designing the pump. When the rotation velocity is increased, the amount of outlet flow is decreased but it has no effect on the amount 
of pump head. When the ratio of inlet liquid is more than that of gas, the flow rate amount is increased while the head amount decreased. The amount of angle of the cylinder pivot with horizon has no effect on flow but when the angle is increased, the head amount decreased. The spiral pitch has no effect on flow and head. The number of the pump coils has no effect on flow but when they are increased, the head increased too. When diameter of the spiral pipe is increased, the flow is increased and the head decreased. Increasing the diameter of the cylinder leads to increasing the flow and head.

\section{References}

1. Cooper P. Analysis of single and two-phase flows in turbopump inducers. Journal for Engineering for Power. 1967; 89577-88.

2 Murakami M, Minemura K. Effect of entrained air on the performance of centrifugal pumps. Bull JSME. 1974; 117:1286-295.

3 Zuchao Z, Peng X, Guofu O, Baoling C, Yi L. Design and experimental analyses of small-flow high-head centrifugal-vortex pump for gas-liquid two-phase mixture. Chin J Chem Eng. 2008; 16(4), 528-34.

4 Shah SR, Jain SV, Patel RN, Lakhera VJ. CFD for centrifugal pumps: a review of the state-of-the-art. Procedia Engineering. 2013; 51:715-20.

5 Pandey KM, Singh AP, Chakrabotry S. Numerical studies on effects of blade number variations on performance of centrifugal pumps AT $2500 \mathrm{rpm}$. Journal of Environmental Research And Development. 2012; 6(3A):863-68.

6 Räbiger K, Maksoud TMA, Ward J, Hausmann G. Theoretical and experimental analysis of a multiphase screw pump, handling gas-liquid mixtures with very high gas volume fractions. Exp Therm Fluid Sci. 2008; 32(8):16941701.

7 Paladino EE, Lima JA, Pessoa PAS, Almeida RFC. A computational model for the flow within rigid stator progressing cavity pumps. Journal of Petroleum Science and Engineering. 2011; 78(1):178-192.

8 Zhang J, Zhu H, Yang C, Yan L, Wei H. Multi-objective shape optimization of helico-axial multiphase pump impeller based on NSGA-II and ANN. Energ Convers Manag. 2010; 52(1):538-46. 